\title{
The uniqueness of invariant measures for Markov operators
}

by

\author{
TOMASz SzareK (Katowice)
}

Dedicated to Professor Jan Kisyński on the occasion of his 75th birthday

\begin{abstract}
It is shown that Markov operators with equicontinuous dual operators which overlap supports have at most one invariant measure. In this way we extend the well known result proved for Markov operators with the strong Feller property by R. Z. Khas'minski.
\end{abstract}

1. Introduction. In this note we are concerned with Markov operators on measures. Our main goal is to prove a criterion for the uniqueness of an invariant measure. The idea of the criterion goes back to R. Z. Khas'minski who showed that every Markov operator satisfying both the strong Feller property and the irreducible condition admits at most one invariant measure [5]. Khas'minski's result is a useful tool in proving the uniqueness of an invariant measure for stochastic differential equations (see [8]). However, the strong Feller property seems to be a restrictive condition, especially for stochastic differential equations with a degenerate noise. We are able to omit this assumption for Markov operators with an equicontinuous dual operator. These operators have been extensively studied by S. P. Meyn and R. L. Tweedie in [7]. We prove that if such operators overlap supports, then they have at most one invariant measure. Let us mention here that the overlapping support property is useful, and indeed essential, for the proof of stability for a large class of Markov operators acting on densities, namely, for Frobenius-Perron operators [1] and partially integral operators [9].

Further, we shall make use of Theorem 3.1 of [6] to show that if the Markov operator under consideration admits a unique invariant measure, then any Markov chain corresponding to it is almost surely dense in the support of the invariant measure. A similar theorem holds for Markov operators which are nonexpansive with respect to the Wasserstein metric (see [6]).

2000 Mathematics Subject Classification: Primary 60J05; Secondary 37A30.

Key words and phrases: Markov operator, invariant measure, dense trajectory. 
If $(X, \varrho)$ is compact, the conclusion is also satisfied for Feller operators. However, it can be shown that Feller operators on general Polish spaces do not have this property (see the counterexample in [6]).

Let $(X, \varrho)$ be a complete and separable metric space. For $x \in X$ and $r>0$ we denote by $B(x, r)$ the open ball centered at $x$ with radius $r$. We denote by $\mathcal{B}(X)$ the spaces of all Borel sets. Further, we denote by $\mathcal{M}=\mathcal{M}(X)$ and $\mathcal{M}_{1}=\mathcal{M}_{1}(X)$ the spaces of all Borel measures and of all Borel probability measures, respectively. Let $\mu \in \mathcal{M}$. We denote by $\operatorname{supp} \mu$ the support of the measure $\mu$, i.e.

$$
\operatorname{supp} \mu=\{x \in X: \mu(B(x, r))>0 \text { for every } r>0\} .
$$

Let $A \in \mathcal{B}(X)$ be such that $\mu(A)>0$. We define $\mu^{A}$ to be $\mu(\cdot \cap A) / \mu(A)$. Obviously $\mu^{A} \in \mathcal{M}_{1}$.

We denote by $B(X)$ the space of all bounded Borel measurable functions. This space is equipped with the supremum norm $\|\cdot\|$. Let $A \subset X$ and $\varepsilon>0$. We set $A^{\varepsilon}=\left\{x \in X: \inf _{y \in A} \varrho(x, y) \leq \varepsilon\right\}$. We will use the scalar product notation:

$$
\langle f, \mu\rangle:=\int_{X} f(x) \mu(d x) \text { for } f \in B(X) \text { and } \mu \in \mathcal{M} .
$$

An operator $P: \mathcal{M} \rightarrow \mathcal{M}$ is called a Markov operator if it satisfies the following conditions:

- $P\left(\lambda_{1} \mu_{1}+\lambda_{2} \mu_{2}\right)=\lambda_{1} P \mu_{1}+\lambda_{2} P \mu_{2}$ for $\lambda_{1}, \lambda_{2} \geq 0, \mu_{1}, \mu_{2} \in \mathcal{M}$;

- $P \mu(X)=\mu(X)$ for $\mu \in \mathcal{M}$.

We say that a Markov operator $P$ overlaps supports if for every $x, y \in X$ there exists $n_{0} \in \mathbb{N}$ such that

$$
\operatorname{supp} P^{n_{0}} \delta_{x} \cap \operatorname{supp} P^{n_{0}} \delta_{y} \neq \emptyset \text {. }
$$

Let $P$ be a Markov operator. An operator $U: B(X) \rightarrow B(X)$ is called dual to $P$ if

$$
\langle f, P \mu\rangle=\langle U f, \mu\rangle \quad \text { for } f \in B(X) \text { and } \mu \in \mathcal{M} .
$$

If the operator $U$ transforms the space $C(X)$ of all bounded continuous functions into itself, then $P$ is called a Feller operator. Finally, if $U$ transforms the space $B(X)$ of all bounded Borel measurable functions into $C(X)$, then $P$ is called strongly Feller.

The function $\pi: X \times \mathcal{B}(X) \rightarrow[0,1]$ given by $\pi(x, A)=P \delta_{x}(A)$ for $x \in X$ and $A \in \mathcal{B}(X)$ is called a transition function.

We denote by $L(X)$ the space of all Lipschitz functions, and by $\operatorname{Lip} f$ the Lipschitz constant of $f \in L(X)$.

The operator $U$ is called equicontinuous if for each $f \in L(X)$ the sequence of functions $\left\{U^{n} f\right\}_{n \geq 1}$ is equicontinuous on compact sets. 
We say that a measure $\mu \in \mathcal{M}$ is invariant with respect to the operator $P$ if $P \mu=\mu$.

A sequence $\left\{\mu_{n}\right\}_{n \geq 1}$ of Borel probability measures is called tight if for each $\varepsilon>0$ there exists a compact set $K \subset X$ such that $\mu_{n}(K)>1-\varepsilon$ for $n \in \mathbb{N}$.

We say that $P$ is recurrent in $D \subset X$ if for every ball $B$ with center at $D$ there is $\alpha>0$ such that

$$
\limsup _{n \rightarrow \infty} P^{n} \delta_{x}(B) \geq \alpha \quad \text { for } x \in D .
$$

2. Invariant measures. The following proposition is crucial for our considerations.

Proposition 2.1. Let $P: \mathcal{M} \rightarrow \mathcal{M}$ be a Feller operator with the equicontinuous dual operator $U$. Let $\mu \in \mathcal{M}_{1}$ be such that the sequence $\left\{P^{n} \mu\right\}_{n \geq 1}$ is tight. Then for each $x \in \operatorname{supp} \mu$ the sequence $\left\{P^{n} \delta_{x}\right\}_{n \geq 1}$ is also tight.

Proof. Let $\mu \in \mathcal{M}_{1}$ be such that the sequence $\left\{P^{n} \mu\right\}_{n \geq 1}$ is tight. Fix $x \in \operatorname{supp} \mu$ and assume, contrary to our claim, that the sequence $\left\{P^{n} \delta_{x}\right\}_{n \geq 1}$ is not tight. Then there exist an $\varepsilon>0$, a sequence $\left\{K_{i}\right\}_{i \geq 1}$ of compact sets and a sequence $\left\{n_{i}\right\}_{i \geq 1}$ of positive integers such that

$$
P^{n_{i}} \delta_{x}\left(K_{i}\right) \geq \varepsilon
$$

and

$$
\min \left\{\varrho(x, y): x \in K_{i}, y \in K_{j}\right\}>\varepsilon \quad \text { for } i \neq j,
$$

by Ulam's lemma ([3], see also the proof of Proposition 2.1 in [11]). We will now define by induction a sequence $\left\{\widetilde{f}_{n}\right\}_{n \geq 1}$ of Lipschitz functions, a sequence $\left\{\nu_{n}\right\}_{n \geq 1}$ of probability measures with supp $\nu_{n} \subset B(x, 1 / n)$, and an increasing sequence $\left\{m_{n}\right\}_{n \geq 0}$ of positive integers with $m_{0}=1$ such that

$$
\begin{gathered}
\widetilde{f}_{n \mid K_{m_{n}}}=1, \quad 0 \leq \widetilde{f}_{n} \leq \mathbf{1}_{K_{m_{n}}^{\varepsilon / 4}}, \quad \operatorname{Lip} \widetilde{f}_{n} \leq 4 / \varepsilon, \\
P^{k} \delta_{x}\left(\bigcup_{i=m_{n}}^{\infty} K_{i}^{\varepsilon / 4}\right) \leq \varepsilon / 8 \quad \text { for } k=1, \ldots, n_{m_{n-1}}, \\
P^{k} \nu_{n}\left(\bigcup_{i=m_{n}}^{\infty} K_{i}^{\varepsilon / 4}\right) \leq \varepsilon / 8 \quad \text { for } k \in \mathbb{N}
\end{gathered}
$$

and

$$
\mid U^{k} f_{n}(x)-U^{k} f_{n}(y) \leq \varepsilon / 4 \quad \text { for } y \in \operatorname{supp} \nu_{n}, f_{n}=\sum_{i=1}^{n-1} \tilde{f}_{i} \text { and } k \in \mathbb{N}
$$


Let $n=1$. Set $A=B(x, 1)$. Since $x \in \operatorname{supp} \mu$, we have $\mu(A)>0$. Let $\nu_{1}=\mu^{A}$. Since $\mu \geq \mu(A) \nu_{1}$ and $\left\{P^{n} \mu\right\}_{n \geq 1}$ is tight, we easily check that $\left\{P^{n} \nu_{1}\right\}_{n \geq 1}$ is also tight. Thus there exists $m_{1} \in \mathbb{N}$ such that (2.3) and (2.4) hold, by (2.2) and the Ulam lemma. Finally, let $\widetilde{f}_{1}$ be an arbitrary continuous function satisfying (2.3) with $m_{n}=m_{1}$.

If $n \geq 1$ is fixed and $\widetilde{f}_{1}, \ldots, \widetilde{f}_{n-1}, \nu_{1}, \ldots, \nu_{n-1}, m_{1}, \ldots, m_{n-1}$ are given, we choose $\delta<1 / n$ such that (2.6) holds for every measure $\nu_{n}$ supported on $B(x, \delta)$ (apply the fact that $\left\{U^{m} f_{n}\right\}_{m \geq 1}$ is equicontinuous at $x$ ). Now set $A=B(x, \delta)$. Let $\nu_{n}=\mu^{A}$ and let $m_{n}>m_{n-1}$ be such that (2.4) and (2.5) are satisfied, by the tightness of $\left\{P^{m} \nu_{n}\right\}_{m \geq 1}$ and $\left\{P^{k} \delta_{x}: k=1, \ldots, n_{m_{n-1}}\right\}$. Finally, let $\widetilde{f}_{n}$ be an arbitrary Lipschitz function satisfying (2.3).

Next, let $f=\sum_{i=1}^{\infty} \widetilde{f}_{i}$. From (2.2) and (2.3) it follows that $f$ is Lipschitzean and $\|f\| \leq 1$. Since $\left\{U^{n} f\right\}_{n \geq 1}$ is equicontinuous at $x$, we have

$$
\left|U^{n_{m_{n}}} f(x)-\left\langle U^{n_{m_{n}}} f, \nu_{n}\right\rangle\right| \rightarrow 0
$$

as $n \rightarrow \infty$, by the fact that $\operatorname{supp} \nu_{n} \subset B(x, 1 / n)$. On the other hand, from (2.1) and (2.3)-(2.5) it follows that

$$
\begin{array}{rl}
\mid U^{n_{m_{n}}} & f(x)-\left\langle U^{n_{m_{n}}} f, \nu_{n}\right\rangle|\geq| U^{n_{m_{n}}} \widetilde{f}_{n}(x)-\left\langle U^{n_{m_{n}}} \widetilde{f}_{n}, \nu_{n}\right\rangle \mid \\
& -\left|U^{n_{m_{n}}}\left(\sum_{i=1}^{n} \widetilde{f}_{i}\right)(x)-\left\langle U^{n_{m_{n}}}\left(\sum_{i=1}^{n} \widetilde{f}_{i}\right), \nu_{n}\right\rangle\right|-\left|U^{n_{m_{n}}}\left(\sum_{i=n+1}^{\infty} \widetilde{f}_{i}\right)(x)\right| \\
& -\left|\left\langle U^{n_{m_{n}}}\left(\sum_{i=n+1}^{\infty} \widetilde{f}_{i}\right), \nu_{n}\right\rangle\right| \geq \varepsilon-\varepsilon / 8-\varepsilon / 4-\varepsilon / 8-\varepsilon / 8=3 \varepsilon / 8,
\end{array}
$$

which is impossible.

Theorem 2.1. Let $P: \mathcal{M} \rightarrow \mathcal{M}$ be a Feller operator with the equicontinuous dual operator $U$. If $P$ overlaps supports, then it admits at most one invariant measure.

Proof. We break up the proof into three steps.

SteP I. Set

$$
\mathbf{T}=\left\{x \in X:\left\{P^{n} \delta_{x}\right\}_{n \geq 1} \text { is tight }\right\} .
$$

To finish the proof it is enough to show that

$$
\lim _{n \rightarrow \infty}\left|U^{n} f(x)-U^{n} f(y)\right|=0 \quad \text { for each } f \in L(X) \text { and } x, y \in \mathbf{T} .
$$

Indeed, suppose, contrary to our claim, that there exist two different measures $\mu_{1}, \mu_{2} \in \mathcal{M}_{1}$ invariant with respect to the operator $P$. Proposition 1.1 
gives supp $\mu_{1}, \operatorname{supp} \mu_{2} \subset \mathbf{T}$. Fix $z \in \mathbf{T}$ and $f \in L(X)$. Then

$$
\begin{aligned}
\left|\left\langle f, \mu_{1}\right\rangle-\left\langle f, \mu_{2}\right\rangle\right|= & \left|\left\langle f, P^{n} \mu_{1}\right\rangle-\left\langle f, P^{n} \mu_{2}\right\rangle\right|=\left|\left\langle U^{n} f, \mu_{1}\right\rangle-\left\langle U^{n} f, \mu_{2}\right\rangle\right| \\
\leq & \left|\left\langle U^{n} f-U^{n} f(z), \mu_{1}\right\rangle\right|+\left|\left\langle U^{n} f-U^{n} f(z), \mu_{2}\right\rangle\right| \\
\leq & \int_{\operatorname{supp} \mu_{1}}\left|U^{n} f(x)-U^{n} f(z)\right| \mu_{1}(d x) \\
& +\int_{\operatorname{supp} \mu_{2}}\left|U^{n} f(x)-U^{n} f(z)\right| \mu_{2}(d x) \quad \text { for } n \in \mathbb{N} .
\end{aligned}
$$

From this and condition (2.8) it follows that $\left\langle f, \mu_{1}\right\rangle=\left\langle f, \mu_{2}\right\rangle$. Since $f \in$ $L(X)$ was arbitrary, we conclude that $\mu_{1} \equiv \mu_{2}$.

SteP II. Fix $x, y \in \mathbf{T}$ and $f \in L(X)$. Fix also $\varepsilon>0$. For $\alpha>0$, set $(\mathbf{T} \times \mathbf{T})_{\alpha}^{\varepsilon}=\left\{\left(x_{1}, x_{2}\right) \in \mathbf{T} \times \mathbf{T}: \exists_{n_{0} \in \mathbb{N}} \exists_{\mu_{1}, \mu_{2} \in \mathcal{M}_{1}} P^{n_{0}} \delta_{x_{i}} \geq \alpha \mu_{i}, i=1,2\right.$, and $\left|\left\langle f, P^{n} \mu_{1}\right\rangle-\left\langle f, P^{n} \mu_{2}\right\rangle\right|<\varepsilon$ for all $\left.n \in \mathbb{N}\right\}$.

Set

$$
\theta:=\sup \left\{\alpha \geq 0:(x, y) \in(\mathbf{T} \times \mathbf{T})_{\alpha}^{\varepsilon}\right\} .
$$

We claim that $\theta=1$. Suppose, contrary to our claim, that $\theta<1$, and let $\left\{\alpha_{k}\right\}_{k \geq 1}$ be such that $\alpha_{k} \uparrow \theta$. Let $n_{k} \in \mathbb{N}$ and $\mu_{1}^{k}, \mu_{2}^{k} \in \mathcal{M}_{1}, k \in \mathbb{N}$, be such that

$$
P^{n_{k}} \delta_{x} \geq \alpha_{k} \mu_{1}^{k} \quad \text { and } \quad P^{n_{k}} \delta_{y} \geq \alpha_{k} \mu_{2}^{k}
$$

Moreover,

$$
\left|\left\langle f, P^{n} \mu_{1}^{k}\right\rangle-\left\langle f, P^{n} \mu_{2}^{k}\right\rangle\right|<\varepsilon \quad \text { for } n \in \mathbb{N} .
$$

Set

$$
\widetilde{\mu}_{1}^{k}=P^{n_{k}} \delta_{x}-\alpha_{k} \mu_{1}^{k} \quad \text { and } \quad \tilde{\mu}_{2}^{k}=P^{n_{k}} \delta_{y}-\alpha_{k} \mu_{2}^{k} .
$$

and observe that the family $\left\{\widetilde{\mu}_{i}^{k}: k \in \mathbb{N}, i=1,2\right\}$ is tight, since $(x, y) \in$ $\mathbf{T} \times \mathbf{T}$. From this and the Prokhorov theorem (see [3]), there exist nontrivial measures $\nu_{1}, \nu_{2} \in \mathcal{M}$ such that some subsequences of the sequences $\left\{\mu_{1}^{k}\right\}_{k \geq 1}$, $\left\{\mu_{2}^{k}\right\}_{k \geq 1}$ (not relabeled) converge weakly to $\nu_{1}, \nu_{2}$, respectively. Choose $v_{1} \in$ $\operatorname{supp} \nu_{1}$ and $v_{2} \in \operatorname{supp} \nu_{2}$ and let $\widetilde{n} \in \mathbb{N}$ be such that

$$
\operatorname{supp} P^{\widetilde{n}} \delta_{v_{1}} \cap \operatorname{supp} P^{\widetilde{n}} \delta_{v_{2}} \neq \emptyset \text {, }
$$

as $P$ overlaps supports. Fix $w \in \operatorname{supp} P^{\widetilde{n}} \delta_{v_{1}} \cap \operatorname{supp} P^{\widetilde{n}} \delta_{v_{2}}$. Since $U$ is equicontinuous, we may choose $r>0$ such that

$$
\left|U^{n} f(w)-U^{n} f(\widetilde{w})\right|<\varepsilon / 2 \quad \text { for } \widetilde{w} \in B(w, r) \text { and } n \in \mathbb{N} .
$$

Set $\gamma=P^{\widetilde{n}} \delta_{v_{1}}(B(w, r)) \wedge P^{\widetilde{n}} \delta_{v_{2}}(B(w, r))$. Since $P$ is a Feller operator, there exists $\tilde{r}>0$ such that

$$
P^{\widetilde{n}} \delta_{v}(B(w, r)) \geq \gamma / 2 \quad \text { for } v \in B\left(v_{1}, \widetilde{r}\right) \cup B\left(v_{2}, \widetilde{r}\right) .
$$


Choose $\beta$ such that $0<\beta<\nu_{1}\left(B\left(v_{1}, \widetilde{r}\right)\right) \wedge \nu_{2}\left(B\left(v_{2}, \widetilde{r}\right)\right)$. As the measures $\widetilde{\mu}_{1}^{k}, \widetilde{\mu}_{2}^{k}$ converge weakly to $\nu_{1}, \nu_{2}$, it follows that $\widetilde{\mu}_{1}^{k}\left(B\left(v_{1}, \widetilde{r}\right)\right) \wedge \widetilde{\mu}_{2}^{k}\left(B\left(v_{2}, \widetilde{r}\right)\right) \geq$ $\beta$ for all sufficiently large $k$, by the Aleksandrov theorem. From (2.13) and the Chapman-Kolmogorov equation we obtain

$$
P^{\widetilde{n}} \widetilde{\mu}_{i}^{k}(B(w, r)) \geq \gamma \beta / 2 \quad \text { for } i=1,2
$$

and large enough $k$. Let $k_{0} \in \mathbb{N}$ be such that the above condition is satisfied and

$$
\alpha_{k_{0}}+\gamma \beta / 2>\theta \text {. }
$$

Define

$$
\widehat{\mu}_{i}(\cdot)=\frac{P^{\widetilde{n}} \widetilde{\mu}_{i}^{k}(\cdot \cap B(w, r))}{P^{\widetilde{n}} \widetilde{\mu}_{i}^{k}(B(w, r))} \quad \text { for } i=1,2
$$

Then we have

$$
\begin{aligned}
& P^{n_{k_{0}}+\widetilde{n}} \delta_{x} \geq \alpha_{k_{0}} P^{\widetilde{n}} \mu_{1}^{k_{0}}+(\gamma \beta / 2) \widehat{\mu}_{1}, \\
& P^{n_{k_{0}}+\widetilde{n}} \delta_{y} \geq \alpha_{k_{0}} P^{\widetilde{n}} \mu_{2}^{k_{0}}+(\gamma \beta / 2) \widehat{\mu}_{2} .
\end{aligned}
$$

From (2.12) and the fact that $\operatorname{supp} \widehat{\mu}_{i} \subset B(w, r), i=1,2$, it follows that

$$
\left|\left\langle f, P^{n} \widehat{\mu}_{1}\right\rangle-\left\langle f, P^{n} \widehat{\mu}_{2}\right\rangle\right|<\varepsilon \quad \text { for } n \in \mathbb{N} .
$$

Set

$$
\begin{aligned}
& \bar{\mu}_{1}=\left(\alpha_{k_{0}}+\gamma \beta / 2\right)^{-1}\left(\alpha_{k_{0}} P^{\widetilde{n}} \mu_{1}^{k_{0}}+(\gamma \beta / 2) \widehat{\mu}_{1}\right), \\
& \bar{\mu}_{2}=\left(\alpha_{k_{0}}+\gamma \beta / 2\right)^{-1}\left(\alpha_{k_{0}} P^{\widetilde{n}} \mu_{1}^{k_{0}}+(\gamma \beta / 2) \widehat{\mu}_{2}\right) .
\end{aligned}
$$

Then condition (2.16) holds with $\bar{\mu}_{1}$ in place of $\widehat{\mu}_{1}$ and $\bar{\mu}_{2}$ in place of $\widehat{\mu}_{2}$, by (2.16) and the definitions of $\mu_{1}^{k_{0}}$ and $\mu_{2}^{k_{0}}$. Finally, from (2.15) it follows that $(x, y) \in(\mathbf{T} \times \mathbf{T})_{\alpha_{k_{0}}+\gamma \beta / 2}$, contrary to (2.14) and the definition of $\theta$.

SteP III. What is left is to show that condition (2.8) holds. Fix an $\varepsilon>0$. We conclude from Step II that $\sup \left\{\alpha \geq 0:(x, y) \in(\mathbf{T} \times \mathbf{T})_{\alpha}^{\varepsilon / 3}\right\}=1$, hence there exist $n_{0} \in \mathbb{N}$ and $\mu_{1}, \mu_{2} \in \mathcal{M}_{1}$ such that

$$
P^{n_{0}} \delta_{x} \geq(1-\varepsilon /(3\|f\|)) \mu_{1} \quad \text { and } \quad P^{n_{0}} \delta_{y} \geq(1-\varepsilon /(3\|f\|)) \mu_{2} .
$$

Moreover,

$$
\left|\left\langle f, P^{n} \mu_{1}\right\rangle-\left\langle f, P^{n} \mu_{2}\right\rangle\right|<\varepsilon / 3 \quad \text { for } n \in \mathbb{N} .
$$

Then an easy calculation shows that

$$
\left|\left\langle f, P^{n+n_{0}} \delta_{x}\right\rangle-\left\langle f, P^{n+n_{0}} \delta_{y}\right\rangle\right|<\varepsilon \quad \text { for } n \in \mathbb{N},
$$

which by Step I finishes the proof.

3. Dense trajectories. Let $P$ be a Feller operator and let $\pi$ be its transition function. It is well known that having the transition function $\pi$ and the space of all Borel probability measure $\mathcal{M}_{1}$, one can construct a probability space $\left(\Omega, \Sigma\right.$, prob) such that for every $\mu \in \mathcal{M}_{1}$ there exists a 
Markov chain $\left\{x_{n}\right\}_{n \geq 0}$ such that $\operatorname{prob}\left(x_{0} \in A\right)=\mu(A)$ and $\operatorname{prob}\left(x_{n+1} \in A \mid\right.$ $\left.x_{n}=x\right)=\pi(x, A)$. We call such a Markov chain corresponding to the Markov operator $P$. The following strengthening of results of [6] holds:

Theorem 3.1. Let $P: \mathcal{M} \rightarrow \mathcal{M}$ be a Feller operator with the equicontinuous dual operator $U$. If $P$ overlaps supports and admits an invariant measure $\mu_{*} \in \mathcal{M}_{1}$, then

$$
\operatorname{prob}\left(\operatorname{cl}\left\{x_{0}, x_{1}, \ldots\right\}=\operatorname{supp} \mu_{*}\right)=1
$$

for every Markov chain $\left\{x_{n}\right\}_{n \geq 0}$ corresponding to $P$ and such that $\operatorname{prob}\left(x_{0} \in\right.$ $\left.\operatorname{supp} \mu_{*}\right)=1$.

Proof. By Theorem 3.1 in [6] it is enough to show that $P$ is recurrent in $\operatorname{supp} \mu_{*}$. Let $B \subset X$ be a ball with center in supp $\mu_{*}$. We will show that condition (1.1) holds with $\alpha=\mu_{*}(B)$. Fix $x \in \operatorname{supp} \mu_{*}$ and consider a sequence $\left\{\mu_{n}\right\}_{n \geq 1}$ of measures given by the formula

$$
\mu_{n}=\frac{1}{n} \sum_{i=1}^{n} P^{i} \delta_{x} \quad \text { for } n \in \mathbb{N} .
$$

We conclude from Proposition 2.1 that the sequence $\left\{P^{n} \delta_{x}\right\}_{n \geq 1}$ is tight and hence so is $\left\{\mu_{n}\right\}_{n \geq 1}$. By Prokhorov's theorem there exists a subsequence $\left\{m_{n}\right\}_{n \geq 1}, m_{n} \rightarrow \infty$ as $n \rightarrow \infty$, such that $\mu_{m_{n}}$ converges weakly to some probability measure $\mu$. Since $\mu$ is invariant with respect to $P$, which, in turn, admits a unique invariant measure $\mu_{*}$, we have $\mu_{*}=\mu$. The Aleksandrov theorem now gives

$$
\liminf _{n \rightarrow \infty} \mu_{m_{n}}(B) \geq \mu_{*}(B),
$$

and consequently

$$
\limsup _{n \rightarrow \infty} P^{n} \delta_{x}(B) \geq \mu_{*}(B) .
$$

Since $x \in \operatorname{supp} \mu_{*}$ was arbitrary, this proves our theorem.

4. Example. We end the paper with an example of a Feller operator $P$ with the equicontinuous dual operator $U$ which does not have the strong Feller property. Recently M. Hairer and J. Mattingly have introduced the class of asymptotic strong Feller operators (see [4]). Observe that these operators also possess an equicontinuous dual operator. We present here a more straightforward example. Our operator will also overlap supports.

Let $P_{1}$ be a strong Feller operator such that supp $P_{1} \delta_{x}=X$ for $x \in X$. We may assume that $P_{1}$ is nonexpansive with respect to the Wasserstein metric, i.e.

$$
d_{W}\left(P_{1} \mu_{1}, P_{1} \mu_{2}\right) \leq d_{W}\left(\mu_{1}, \mu_{2}\right) \quad \text { for all } \mu_{1}, \mu_{2} \in \mathcal{M}_{1},
$$


where

$$
d_{W}\left(\mu_{1}, \mu_{2}\right)=\sup \left\{\left|\left\langle f, \mu_{1}\right\rangle-\left\langle f, \mu_{2}\right\rangle\right|: \operatorname{Lip} f \leq 1 \text { and }\|f\| \leq 1\right\} .
$$

In the case when $X$ is a Hilbert space, $P_{1}$ may be defined as a Markov operator corresponding to some stochastic differential equation (see [2]).

Let $P_{2}$ be an arbitrary nonexpansive Markov operator with respect to the Wasserstein norm operator. Then $P_{2}$ is a Feller operator (see [10]). We assume that $P_{2}$ is not strongly Feller. Such an operator may be defined as a Markov operator associated with some iterated function system (see [11]). Define a new Markov operator by

$$
P=P_{1} / 2+P_{2} / 2 .
$$

Observe that $P$ is nonexpansive with respect to the Wasserstein metric. Further, since supp $P_{1} \delta_{x}=X$ for $x \in X$, we have supp $P \delta_{x} \cap \operatorname{supp} P \delta_{y} \neq \emptyset$ for $x, y \in X$. On the other hand, $P$ is not strongly Feller. Finally,

$$
\begin{aligned}
d_{W}\left(P^{n} \delta_{x}, P^{n} \delta_{y}\right) & =\sup \left\{\left|U^{n} f(x)-U^{n} f(y)\right|: \operatorname{Lip} f \leq 1,\|f\| \leq 1\right\} \\
& \leq d_{W}\left(\delta_{x}, \delta_{y}\right) \leq \varrho(x, y) \quad \text { for } n \in \mathbb{N},
\end{aligned}
$$

which shows that $U$ is equicontinuous. From Theorem 2.1 it follows that $P$ admits at most one invariant measure.

Acknowledgements. I would like to express my gratitude to the late Professor Andrzej Lasota who both inspired and encouraged me with this study. Without his kind words and many long and stimulating discussions this research would not have been done. The preparation of this paper was supported by the European Commision Marie Curie Host Fellowship for the Transfer of Knowledge "Harmonic Analysis, Nonlinear Analysis and Probability" MTKD-CT-2004-013389. I also thank the University of Wrocław and especially P. Biler and G. Karch for their warm hospitality during the Spring Semester 2007. This work was also partly supported by Polish Ministry of Science and Higher Education Grant N N201 021133.

\section{References}

[1] W. Bartoszek and T. Brown, On Frobenius-Perron operators which overlap supports, Bull. Polish Acad. Sci. Math. 45 (1997), 17-24.

[2] G. Da Prato and J. Zabczyk, Stochastic Equations in Infinite Dimensions, Cambridge Univ. Press, Cambridge, 1992.

[3] S. N. Ethier and T. G. Kurtz, Markov Processes: Characterization and Convergence, Wiley, New York, 1985.

[4] M. Hairer and J. Mattingly, Ergodicity of the 2D Navier-Stokes equations with degenerate stochastic forcing, Ann. of Math. 164 (2006), 993-1032.

[5] R. Z. Khas'minski, Ergodic properties of recurrent diffusion processes and stabilization of the solution to the Cauchy problem for parabolic equations, Theor. Probab. Appl. 5 (1960), 179-196. 
[6] A. Lasota, J. Myjak and T. Szarek, Markov operators with a unique invariant measure, J. Math. Anal. Appl. 276 (2002), 343-356.

[7] S. P. Meyn and R. L. Tweedie, Markov Chains and Stochastic Stability, Springer, London, 1993.

[8] S. Peszat and J. Zabczyk, Strong Feller property and reducibility for diffusions on Hilbert spaces, Ann. Probab. 23 (1995), 157-172.

[9] R. Rudnicki, On asymptotic stability and sweeping for Markov operators, Bull. Polish Acad. Sci. Math. 43 (1995), 245-262.

[10] T. Szarek, Invariant measures for nonexpansive Markov operators on Polish spaces, Dissertationes Math. 415 (2003).

[11] —, Feller processes on non-locally compact spaces, Ann. Probab. 34 (2006), 1849 1863.

Institute of Mathematics

Silesian University

Bankowa 14

40-007 Katowice, Poland

E-mail: szarek@intertele.pl

Received July 20, 2007

Revised version June 2, 2008 